\title{
Tachycardia Detection in Implantable Antitachycardia Devices
}

\author{
JANICE JENKINS, * THOMAS BUMP, FRAN MUNKENBECK, JEFFREY \\ BROWN, and ROBERT ARZBAECHER \\ From the Illinois Institute of Technology and University of Chicago, Chicago, Illinois, and *The \\ University of Michigan, Ann Arbor, Michigan USA
}

La détection des tachycardies par les stimulateurs antitachycardie implantables. Nous avons développé un algorithme permettant l'identification des tachycardies par un microcomputer utilisable dans un pacemaker antitachycardie. Celui ci distingue les tachycardies auriculaires, ventriculaires et de réentrée du noeud $A V$ et ne reconnait pas les tachycardies sinusales. De même a été développé une nouvelle méthode permettant au pacemaker à l'aide d'un extrastimulus auriculaire de distinguer les tachycardies paroxystiques 1/1 des tachycardies sinusales. Celle ci est actuellement testée au laboratoire d'électrophysiologie. Les résultats préliminaires indiquent que ces deux tachycardies peuvent être distinguées de façon fiable par un extrastimulus délivré avec un couplage correct dans l'oreillette droite.

JENKINS, J., BUMP, T., MUNKENBECK, F., ET AL.: Tachycardia detection in implantable antitachycardia devices. We have developed a microcomputer algorithm for tachycardia identification suitable for use in an implanted antitachycardia pacemaker. The algorithm distinguishes atrial, ventricular, and $A V$ reentrant tachycardia, while ignoring high rates due to sinus tachycardia. A new method whereby the pacemaker delivers an atrial extrastimulus has been developed for distinguishing paroxysmal 1:1 tachycardia from sinus tachycardia, and has undergone preliminary testing in the electrophysiology laboratory. Preliminary results indicate sinus tachycardia can be reliably distinguished from paroxysmal 1:1 tachycardia by a properly timed extrastimulus delivered to the right atrium.

tachycardias, detection algorithms

\section{Introduction}

The development of antitachycardia pacemakers designed for conversion of malignant tachycardias has created a demand for more accurate tachycardia recognition schemes than those presently available. Fortunately, rate microelectronic technology now provides the mechanism for incorporating a microprocessor within the pacemaker, and thus computer pattern recognition schemes can be included within the device. This paper describes an algorithm we have developed that is suitable for use within such a microprocessor-based antitachycardia pacemaker. The algorithm distinguishes atrial, ventricular, and AV

Address for reprints: Janice Jenkins, Ph.D., Department of Electrical Engineering and Computer Science, The University of Michigan, Ann Arbor, MI 48109 USA reentrant tachycardia and uses a criterion of rapid onset to distinguish pace-terminable tachycardias from sinus tachycardia.

Antitachycardia pacemakers that are at present undergoing clinical testing detect tachycardia by sensing a high rate in the chamber to be paced. The rate must exceed a preset threshold and the tachycardia must persist for a preselected number of beats. Such simple criteria may result in inaccurate identification of a tachycardia and result in undesirable pacing. Other schemes ${ }^{1-3}$ have been advanced for using criteria other than rate but most have not yet been tested.

There is a particular problem with sinus tachycardia which is often indistinguishable from paceterminable 1:1 tachycardias on the basis of rate alone, and these onset measures have been proposed. It is commonly held that sinus tachycardias accelerate gradually while pace-terminable tachycardias are sudden in onset. However, exceptions 
do occur since sinus tachycardia resulting from fright, excitement, sudden effort, or postural change can occasionally develop very quickly, while paroxysmal tachycardias may begin quite hesitantly, with couplets and triplets separated by long pauses. Also, premature beats and their compensatory pauses can occur just before or just after the beginning of a tachycardia. Thus a successful algorithm for assessing suddenness of onset must examine more than just the two intervals which define the transition from below the tachycardia rate threshold to above it.

One newly developed algorithm has decision rules for measuring the suddenness of onset for purposes of separating sinus tachycardia from paroxysmal tachycardia. This algorithm, called INTERTACH was developed for the antitachycardia

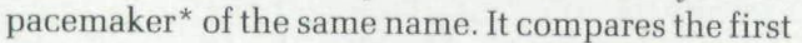
interval of the tachycardia run (the first to fall below the tachycardia threshold) to the first previous (pre-tach) cycle length, two cycle lengths just preceding the tachycardia. If the first interval in the tachycardia is shorter than either by a preset amount (and if the third interval preceding the tachycardia is not short) the tachycardia is paroxysmal; otherwise, it is sinus. The algorithm performs reasonably well in discriminating sinus from pace-terminable tachycardias. Nevertheless, there are paroxysmal tachycardias that start more slowly than sudden sinus tachycardias and these cannot always be separated by onset criteria alone. Fischer et al. ${ }^{4}$ examined 50 spontaneous ventricular tachycardias and 50 sinus tachycardias and found that while the rate of tachycardia development is significantly faster with ventricular tachycardia, some overlap still exists for individual subjects.

In addressing the above problem, we have developed our own onset criterion and have also explored an extrastimulus test, performed by the pacemaker itself, to identify sinus tachycardia. In sinus tachycardia, the ventricular rhythm is perturbed by the atrial extrastimulus. In the other rhythms shown, the ventricular response is not perturbed by the extrastimuli.

\section{Methods}

Our computer algorithm for tachycardia recognition is shown in Figure 1. Full details have been

${ }^{\star}$ Intermedics, Freeport, Texas, USA

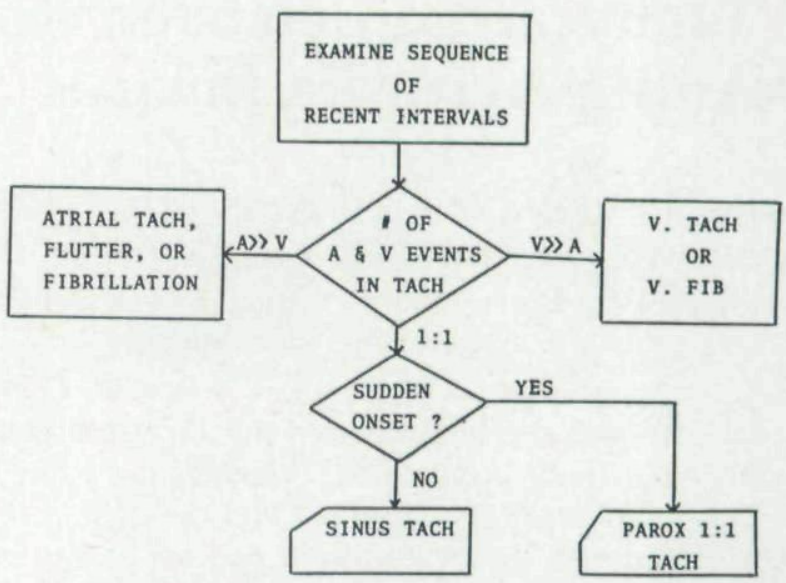

Figure 1. Flow chart of the main diagnostic program. Dual-chamber sensing allows separation of atrial and ventricular tachycardia, and the analysis of onset distinguishes tachycardia.

previously published. ${ }^{5}$ Following the detection of a sustained run of short intervals in either chamber, the program determines whether the events in the tachycardia are predominantly atrial or ventricular. If they are atrial, the left path of the flow chart is followed and atrial tachycardia, flutter, or fibrillation is diagnosed, depending on rate. If events are predominantly ventricular, the right path is taken and ventricular tachycardia or fibrillation is diagnosed. If the number of atrial and ventricular events is roughly equal, 1:1 tachycardia is diagnosed, and a measure of suddenness of onset is made in order to distinguish ordinary sinus tachycardia from other 1:1 tachycardias: atrial, AV reentrant, AV nodal reentrant, and ventricular with retrograde conduction to the atria. In this test, the first four beats that satisfy the high rate condition that invoke the program are compared to the four beats preceding them. If they have an interbeat interval $25 \%$ shorter than the average of the preceding four, the tachycardia is paroxysmal and presumably pace-terminable; otherwise, the tachycardia is sinus tachycardia.

For evaluation of the algorithm, we selected 22 electrically induced tachycardias from patients whose electrograms were tape recorded during intracardiac electrophysiologic study. All recordings contain at least three surface and four bipolar catheter leads, including electrograms from the right atrium, right ventricle, bundle of His, and coronary sinus. Only the atrial and ventricular elec- 
trograms were used by the computer, but all were available to the electrophysiologist, who independently diagnosed each recording for comparison with the computer diagnosis.

Following evaluation of the above-described algorithm, and motivated by a concern that gradual onset may not be a completely sensitive and specific test for sinus tachycardia, we developed and tested an extrastimulus technique for confirming the diagnosis of sinus versus pace-terminable 1:1 tachycardias. In this new concept, we hypothesized that a premature extra stimulus would result in a premature ventricular response during sinus tachycardia but not during many forms of 1:1 paceterminable tachycardias. If a tachycardia is ventricular, AV reentrant, or AV nodal reentrant, the wavefront of the stimulated atrial extrasystole will collide with the retrogradely conducted impulse of the tachycardia and the rhythm of the ventricle will not be perturbed. This is illustrated in Figure 2 . The ladder diagram represents the spontaneous rhythm in which a stimulated atrial extrasystole and its propagated wavefront is imposed. The broken line represents what would have depolarized if the atrial extrastimulus had not occurred.

Eleven patients were tested during electrophysiologic studies to evaluate our concept for automatic atrial extrastimulus testing during

ATFUL EXTRASTMULATON DUFWV TACHYCAROU
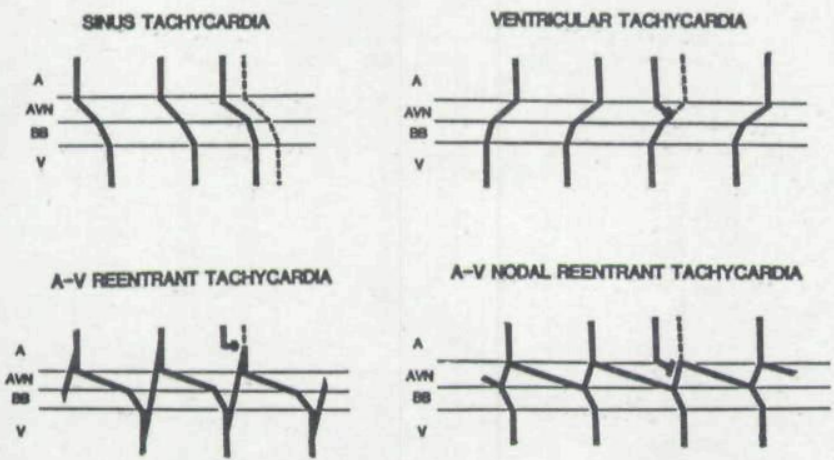

Figure 2. Ladder diagrams representing a spontaneous rhythm in which a hypothetical stimulated atrial extrasystole is introduced. The broken line represents what would have depolarized if the atrial extrastimulus had not occurred. The premature extra stimulus will result in a premature ventricular response during sinus tachycardia but not during many forms of $1: 1$ paceterminable tachycardias. tachycardia. Seven patients had 10 different 1:1 paroxysmal tachycardias. There were 5 orthodromic AV reentrant tachycardias all using leftsided bypass tracts, 3 AV nodal reentrant tachycardias, and 2 atrial tachycardias. Seven of these 11 were tested during sinus tachycardia (induced by atropine and/or isoproterenol) as well as during their pace-terminable tachycardia. Three patients had both sinus tachycardia and their respective paroxysmal 1:1 tachycardias. Recording electrodes were placed in the high right atrium and right ventricular apex. After induction and stabilization of the tachycardia, single extrastimuli were delivered to the high right atrium beginning at the end of diastole and decrementing by $10 \mathrm{~ms}$ until atrial refractoriness was encountered. The ventricular response was carefully measured from 100 $\mathrm{mm} / \mathrm{s}$ strip-chart recordings and AV conduction curves were drawn.

\section{Results}

Twenty-two tape recorded electrograms were processed automatically by the computer for tachycardia recognition and 21 were diagnosed correctly. These included: atrial fibrillation (2), atrial flutter (2), atrial tachycardia (2), ventricular tachycardia (4), 1:1 paroxysmal tachycardia (9), and sinus tachycardia (3). The single error occurred in diagnosing an almost 1:1 ventricular tachycardia with retrograde activation and occasional ventriculoatrial block. Figure 3 shows an example of an AV nodal tachycardia which was initiated by two atrial stimuli. The atrial premature beats conduct with delay to the ventricles and initiate paroxysmal AV nodal tachycardia with a cycle length of $350 \mathrm{~ms}$. The tachycardia has oneto-one atrioventricular relationship and is diagnosed correctly by the computer algorithm as paroxysmal 1:1 tachycardia.

The atrial extra stimulus method was tested on 11 patients. Increasingly early atrial extrastimuli caused the subsequent ventricular beat to be more premature in all 7 patients with sinus tachycardia and in none of the 10 patients with paroxysmal $1: 1$ tachycardias. In particular, when the atrial extrastimulus was premature by $80 \mathrm{~ms}$, the subsequent ventricular response was at least $30 \mathrm{~ms}$ early in all 7 patients with sinus tachycardia and less 


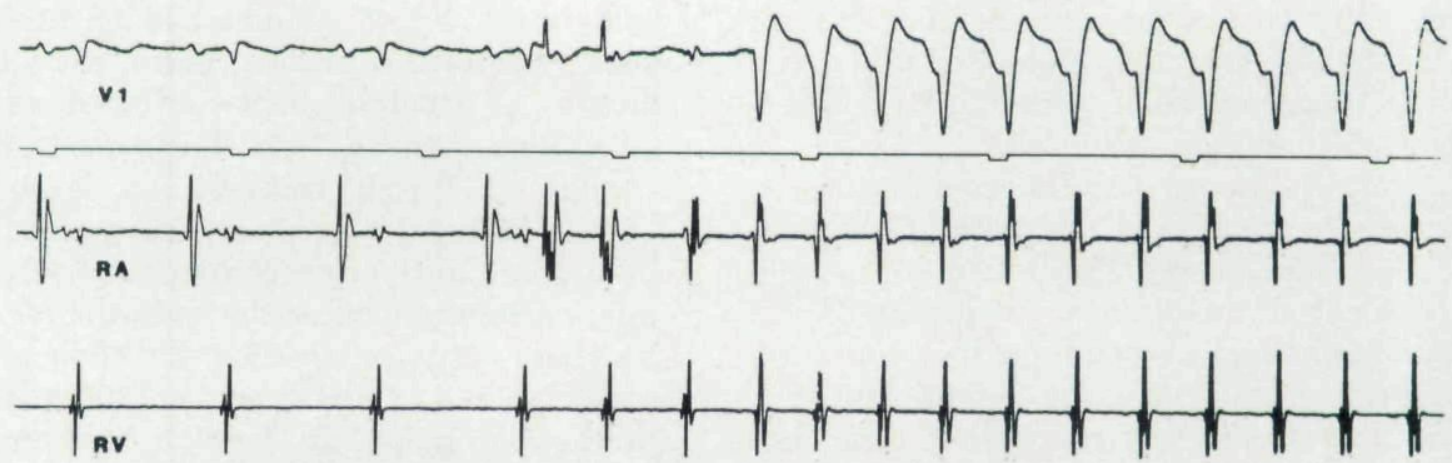

Figure 3. The first four complexes are sinus beats with the cycle length of $780 \mathrm{~ms}$ followed by two atrial extrastimuli that capture the atrium. The atrial premature beats conduct with delay to the ventricles and initiate paroxysmal AV nodal tachycardia at the cycle length of $350 \mathrm{~ms}$. The first complex is narrow and subsequent complexes are aberrantly conducted with LBBB configuration. The tachycardia has one to one atrio-ventricular conduction. (Computer: paroxysmal 1:1 tachycardia)

AES DURING TACHYCARDIA
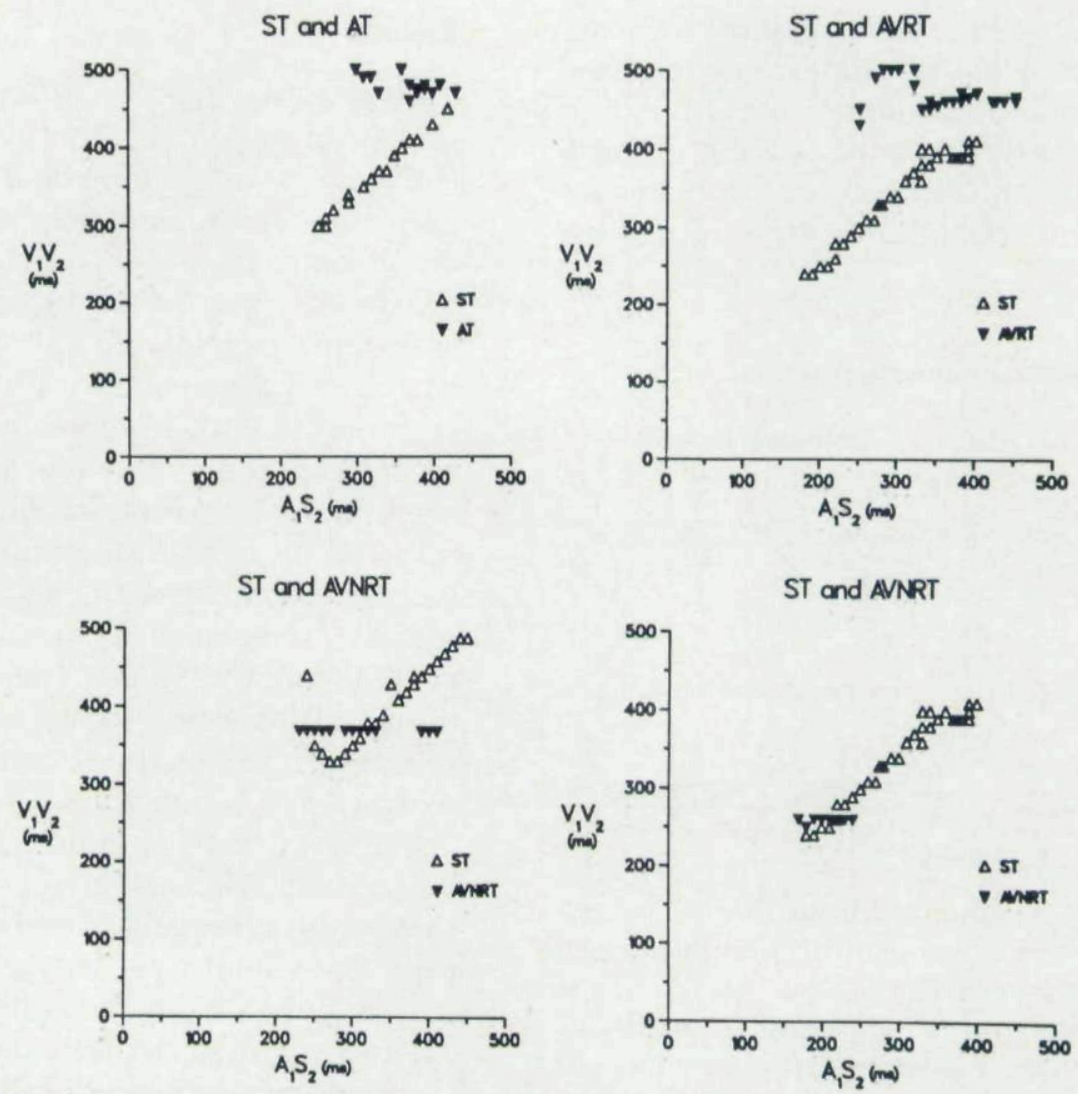

Figure 4. Conduction curves obtained during both the patient's sinus tachycardia and paroxysmal tachycardia in four cases. Open triangles represent results obtained during sinus tachycardia and solid triangles represent data obtained during paroxysmal 1:1 tachycardia. 
than or equal to $10 \mathrm{~ms}$ early in all 10 cases of paroxysmal tachycardia. When the atrial extrastimulus was premature by $100 \mathrm{~ms}$, the next ventricular beat was at least $50 \mathrm{~ms}$ premature in all 7 patients with sinus tachycardia and less than or equal to $10 \mathrm{~ms}$ premature in all 10 cases of paroxysmal 1:1 tachycardia.

Figure 4 presents data from four cases. Each graph shows conduction curves obtained during both the patient's sinus tachycardia and his paroxysmal 1:1 tachycardia. Results obtained during sinus tachycardia are indicated by the open triangles and those obtained during paroxysmal 1:1 tachycardia are indicated by the solid triangles. In sinus tachycardia, as the atrial extrastimulus becomes more premature the ventricular response becomes increasingly premature in all panels of Figure 4. In atrial tachycardia (upper left), the ventricular response does not become more premature when the atrial extrastimulus becomes more premature. The same phenomenon is seen in the case of AV reentrant tachycardia (upper right) and in two cases of AV nodal reentrant tachycardia (lower left and right). Single atrial extrastimuli during tachycardia did not provoke faster tachycardias in any of our patients, but did terminate one paroxysmal tachycardia.

\section{References}

1. Camm, A.J., Nathan, A.W., Ward, D.E.: Responsive pacing for tachycardia termination. Vectors, 9:2, 1981.

2. Bexton, R., Ward, D.E., Hellestrand, K., et al.: QT interval during paroxysmal functional tachycardia. Eur. Heart J., 2:186, 1981.

3. Furman, S., Fischer, J.D., Pannizo, F.: Necessity of signal processing in tachycardia detection. In S.S. Barold and J. Mugica (Eds.): The Third Decade of Cardiac Pacing, New York, Futura Publishing Company, 1982, p. 265.

\section{Conclusions}

We have developed dual-chamber recognition schemes that differentiate among most types of tachycardias and have demonstrated accuracy in $21 / 22$ tachycardias. In an effort to further refine the distinction of sinus from paroxysmal 1:1 tachycardia, we have tested an additional concept in which we deliver an atrial extrastimulus and examine the relationship of ventricular response. In all cases of sinus tachycardia the premature atrial stimulus evoked a similar premature ventricular response. In all cases of paroxysmal tachycardia the premature atrial stimulus led to no ventricular response more premature than $10 \mathrm{~ms}$.

It must be pointed out that interpretation of the response to the atrial extrastimulus would be difficult if the tachycardia were not regular. Also the method may fail to distinguish sinus tachycardia from AV reentrant tachycardia using a right-sided pathway or from the uncommon form of AV nodal tachycardia. With these reservations we believe the atrial extrastimulus shows promise in safely differentiating sinus tachycardia from paroxysmal $1: 1$ tachycardia in most patients and would prove useful in an antitachycardia device. Further evaluation of the method in a larger number of patients with a wider variety of tachycardias is indicated.

4. Fischer, J.D., Goldstein, M., Ostrow, E., et al.: Maximal rate of tachycardia development: Sinus tachycardia with sudden exercise vs. spontaneous ventricular tachycardia. PACE, 6:221, 1983.

5. Arzbaecher, R., Bump, T., Jenkins, J., et al.: Automatic tachycardia recognition. PACE, 7, 1984 (in press). 
Copyright of Pacing \& Clinical Electrophysiology is the property of Blackwell Publishing Limited and its content may not be copied or emailed to multiple sites or posted to a listserv without the copyright holder's express written permission. However, users may print, download, or email articles for individual use. 